\title{
Notes on style
}

Terms in German have been translated and explained when they have been first used; most such terms are also included in the glossary. In a few cases, German terms have been utilised throughout the book, for example for the chambers of the federal legislature (the Bundestag and Bundesrat), for the states that form the Federation (the Länder), and for the parliamentary party group (the Fraktion).

Translations from German sources are the responsibility of the author. The original text is offered in a footnote. 